\title{
Reliable keratometry with a new hand held surgical keratometer: calibration of the keratoscopic astigmatic ruler
}

\author{
Nigel Morlet, Anthony Maloof, Nicholas Wingate, Peter Lindsay
}

Department of Ophthalmology, Prince of Wales Hospital, UNSW, Sydney, Australia N Morlet

A Maloof

Moorfields Eye

Hospital, London

N Morlet

$\mathrm{N}$ Wingate

SVRC, University

Queensland, Brisbane,

Australia

P Lindsay

Correspondence to:

Nigel Morlet, 592 Stirling

Highway Mosman Park 6012

WA Australia

Accepted for publication 18 September 1997

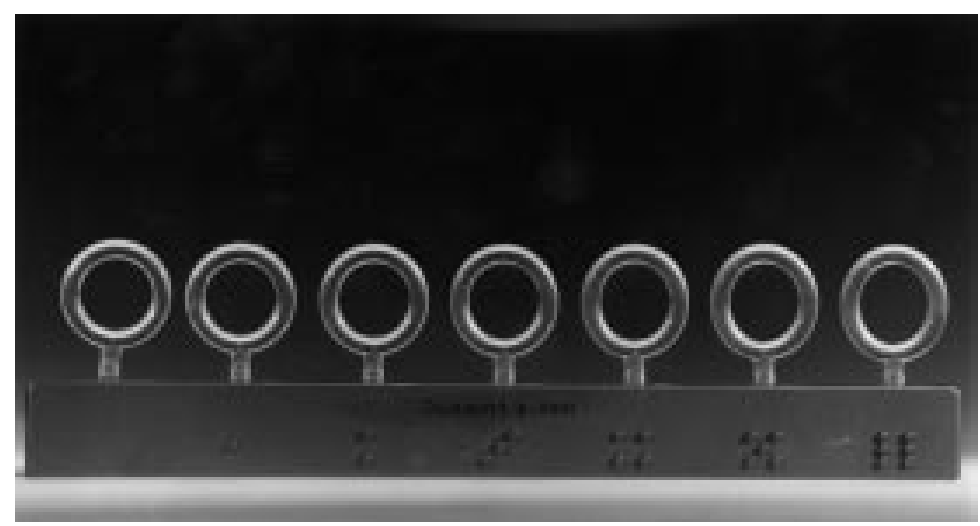

Figure 1 The keratoscopic astigmatic ruler consists of a rack of lenses-a circular lens and six increasingly elliptical lenses for 1 to 6 dioptres of astigmatism. Each keratoscopic lens works like a Maddox rod producing a bright aerial image of the microscope's light that is reflected back to the observer by the corneal surface. The metal handle has raised marks to enable the user to determine, by palpation, the lens in use when at the microscope. The circular lens is used for the initial assessment of the astigmatism and to align the handle of the KAR with the long axis of the elliptical image. The correct distance which hold the KAR from the eye is determined by observing the whole limbal diameter just within the short internal diameter of the lens (the "sight"). Once aligned the image is observed with each lens in front of the cornea, the measurement end point is when the image appears most circular. Past the end point the image becomes elliptical perpendicular to the handle. measurement for the KAR was $0.59 \mathrm{D}$, for the B\&L, 0.31 D. The intraclass correlation coefficient of reliability for the KAR was 0.90 and for the $B \& L$ it was 0.97 . The coefficient of repeatability for the KAR was plus or minus $0.83 \mathrm{D}$, and for the $B \& L$ plus or minus $0.77 \mathrm{D}$. The interobserver reliability for the KAR was $\mathbf{0 . 8 9 8}$, and for the B\&L, 0.975 .

Conclusion-These results suggest that the KAR has good reliability and reproducibility and compares favourably with the $B \& L$ keratometer. Inexperience with use does not affect reliability.

(Br F Ophthalmol 1998;82:35-38)

Determining the magnitude of corneal astigmatism with hand held keratoscopes is considered unreliable by some surgeons. ${ }^{1}$ Difficulties in attaining a corneal image with devices that only use the reflection of the microscope's light may produce artefacts owing to the need to tilt the device to attain a good image. As most devices are unable to quantify the magnitude of astigmatism, an assessment of astigmatism depends upon determining how elliptical the image appears. We have shown that the elliptical astigmatic image becomes more circular as the device is held closer to the eye and that this change in the shape of the image can lead to the underestimation of astigmatism, particularly with higher magnitudes of astigmatism. ${ }^{23}$

The design of keratoscopic astigmatic ruler (KAR) (Fig 1) addresses these problems by producing a bright easily determined image, having a simple method of alignment which sets the distance at which it is held (a "sight"), and allowing the magnitude of astigmatism to be assessed easily. ${ }^{3}$

This report demonstrates that the assessment of astigmatism by the KAR is reliable and accurate, comparing favourably with the Bausch \& Lomb keratometer (B\&L) a conventional clinical keratometer.

\section{Methods}

We fabricated a "model cornea" using a series of poly(methylmethacrylate) toric backcut contact lens blanks (buttons). Six buttons were mounted on a backing plate representing a series of increasing astigmatism in roughly 1 dioptre (D) steps.

Two observers made measurements of the buttons using the KAR and the B\&L keratometer. Observer 1 had no previous experience with the KAR whereas observer 2 was an 
Table 1 Mean and standard deviations (SD) of five measurements of the distance $(\mathrm{mm})$ that the keratoscopic astigmatic ruler was held from the corneal apex of five patients by two observers (observer 1 was a novice, observer 2 an experienced user)

\begin{tabular}{llllllll}
\hline & \multicolumn{3}{l}{ Observer 1} & & & \multicolumn{3}{l}{ Observer 2} \\
\cline { 2 - 3 } \cline { 6 - 8 } Patient & Mean & SD & Min-max & & Mean & SD & Min-max \\
\hline 1 & 20.8 & 1.3 & $20-23$ & & 19.5 & 1.5 & $17-21$ \\
2 & 20.6 & 1.1 & $19-22$ & & 20.3 & 0.5 & $20-21$ \\
3 & 19.8 & 1.3 & $18-21$ & & 19.8 & 0.6 & $19-20.5$ \\
4 & 21.0 & 1.0 & $20-22$ & & 20.0 & 1.0 & $19-21$ \\
5 & 19.8 & 1.8 & $17-22$ & & 20.3 & 0.5 & $20-21$ \\
Overall & 20.4 & 1.3 & & & 20.0 & 0.9 & \\
\hline
\end{tabular}

No significant difference between observers $(p=0.499$, ANOVA)

Table 2 (A) The mean and standard deviation (SD) of eight measurements of the astigmatism (in dioptres) of six toric backcut contact lens blanks (buttons) by two observers using the keratoscopic astigmatic ruler (observer 1 was a novice, observer 2 an experienced user)

\begin{tabular}{llllll}
\hline & \multicolumn{2}{l}{ Observer 1} & & \multicolumn{2}{l}{ Observer 2} \\
\cline { 2 - 3 } \cline { 5 - 6 } Button & Mean & $S D$ & & Mean & $S D$ \\
\hline 1 & 1.125 & 0.354 & & 1.0 & 0 \\
2 & 2.0 & 0.535 & & 2.75 & 0.463 \\
3 & 3.125 & 0.641 & & 3.0 & 0.756 \\
4 & 4.25 & 0.707 & & 4.125 & 0.835 \\
5 & 5.25 & 0.463 & & 4.75 & 0.707 \\
6 & 5.75 & 0.707 & & 6.0 & 0 \\
\hline
\end{tabular}

No significant difference between observers ( $\mathrm{p}=1.0$, ANOVA).

(B) The mean and standard deviation (SD) of four measurements of the astigmatism of six toric backcut contact lens blanks (buttons) by two observers using the Bausch E Lomb keratometer (observer 1 was a novice, observer 2 an experienced user)

\begin{tabular}{llllll}
\hline & \multicolumn{2}{l}{ Observer 1} & & \multicolumn{2}{l}{ Observer 2} \\
\cline { 2 - 3 } \cline { 5 - 6 } Button & Mean & $S D$ & & Mean & $S D$ \\
\hline 1 & 0.312 & 0.217 & & 0.625 & 0.27 \\
2 & 1.406 & 0.062 & & 1.656 & 0.213 \\
3 & 2.594 & 0.188 & & 3.125 & 0.102 \\
4 & 3.531 & 0.461 & & 3.781 & 0.062 \\
5 & 4.875 & 0.177 & & 4.769 & 0.208 \\
6 & 5.219 & 0.258 & & 5.95 & 0.316 \\
\hline
\end{tabular}

No significant difference between observers $(p=0.953$, ANOVA).

Intraclass correlation coefficient of reliability (between observers): KAR, R = 0.898 (95\% CI >0.781), B\&L, R = $0.975(95 \%$ CI >0.633)

Coefficient of variation: $\mathrm{KAR}=0.073, \mathrm{~B} \& \mathrm{~L}=0.094$

Table 3 Analysis of variance of the means and standard deviations (SD) of multiple measurements of the six toric backcut contact lens blanks (buttons) using the Bausch $\mathcal{E}$ Lomb keratometer (BE $L$ ) and keratoscopic astigmatic ruler (KAR) (dioptres)

\begin{tabular}{|c|c|c|c|c|c|c|}
\hline \multirow[b]{2}{*}{ Button } & \multicolumn{3}{|c|}{$K A R$} & \multicolumn{3}{|c|}{$B \mathcal{E} L$} \\
\hline & $N o$ & Mean & $S D$ & No & Mean & $S D$ \\
\hline 1 & 16 & 1.063 & 0.25 & 8 & 0.469 & 0.281 \\
\hline 2 & 16 & 2.375 & 0.619 & 8 & 1.531 & 0.198 \\
\hline 3 & 16 & 3.063 & 0.68 & 8 & 2.859 & 0.316 \\
\hline 4 & 16 & 4.188 & 0.75 & 8 & 3.656 & 0.332 \\
\hline 5 & 16 & 5.0 & 0.632 & 8 & 4.822 & 0.187 \\
\hline 6 & 16 & 5.875 & 0.5 & 8 & 5.584 & 0.473 \\
\hline
\end{tabular}

Standard error of measurement: $\mathrm{KAR}=0.59 \mathrm{D}, \mathrm{B} \& \mathrm{~L}=0.31 \mathrm{D}$. Intraclass correlation coefficient of reliability: KAR, $\mathrm{R}=0.90$ (95\% CI >0.79), B\&L, R = 0.97 (95\% CI >0.94)

Coefficient of variation: $\mathrm{KAR}=0.405, \mathrm{~B} \& \mathrm{~L}=0.091$

Coefficient of repeatability ( $95 \% \mathrm{CI}$ test-retest): $\mathrm{KAR}= \pm 0.83$ $\mathrm{D}, \mathrm{B} \& \mathrm{~L}= \pm 0.77 \mathrm{D}$

experienced user. Both had experience with the use of the B\&L keratometer.

Because the concave surface gives a different sized elliptical image than a convex reflecting

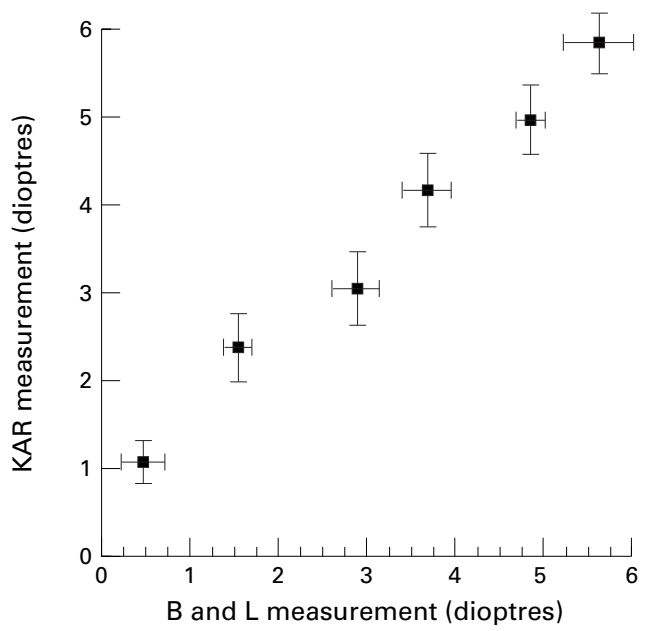

Figure 2 Measurement confidence limits (95\%) of the keratoscopic ruler and the Bausch $\mathcal{E}$ Lomb keratometer

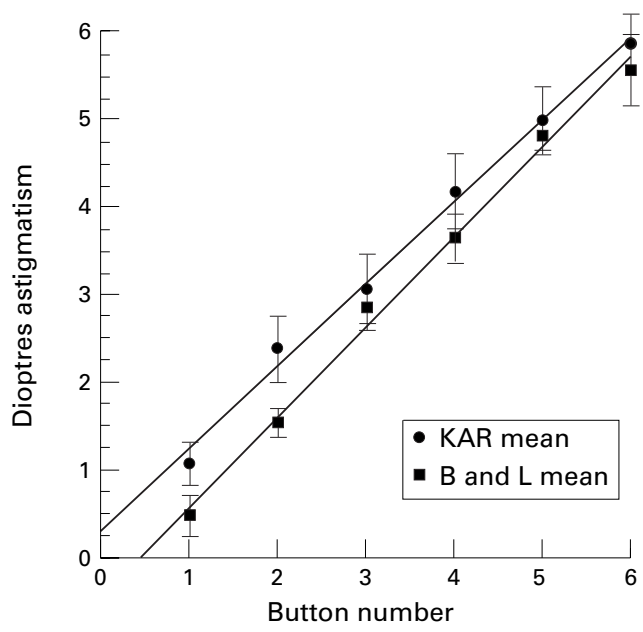

Figure 3 Measurement of the astigmatic concave buttons using the Bausch $\mathcal{E}$ Lomb keratometer and the keratoscopic astigmatic ruler (95\% CI)

surface, we had to hold the KAR further from the elliptical surface than the calibrated distance for clinical use. To ensure the distance was held correctly (because there was no limbus to observe through the sight of the KAR), we placed a stop at the required distance, ensuring that the KAR was not held too close to the button during measurement.

The buttons were placed under the operating microscope in a predetermined randomised order in such a way that the observer was masked to the magnitude and axis of astigmatism. Each button was measured by each of the two observers eight times. The astigmatism was assessed in the same manner on each occasion as described by the instructions packaged with the KAR. First the axis of astigmatism was determined and the handle of the instrument aligned correctly using the circular lens. Then the magnitude of astigmatism was determined once a circular image was produced by one of the elliptical lenses. ${ }^{3}$

The buttons were then measured using a previously calibrated $\mathrm{B} \& \mathrm{~L}$ keratometer ${ }^{4}$ after setting the eye piece to match the refraction of the observer and using the recommended 


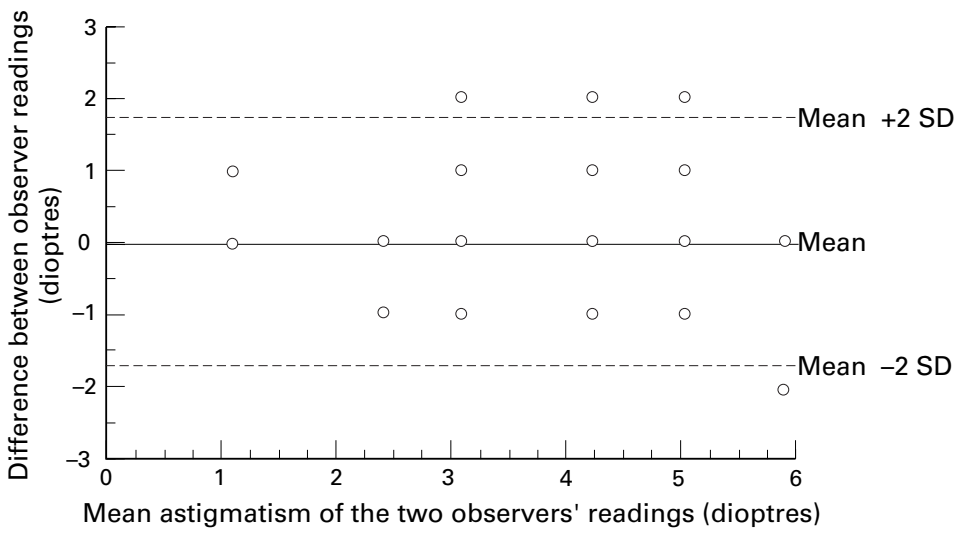

Figure 4 Limits of agreement between observers using the keratoscopic astigmatic ruler.

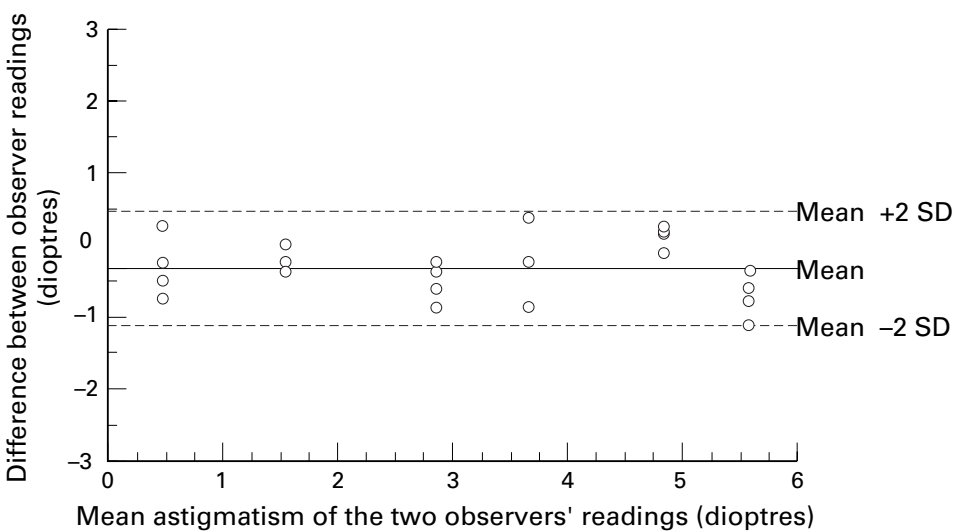

Figure 5 Limits of agreement between observers using the Bausch $\mathcal{E}$ Lomb keratometer.

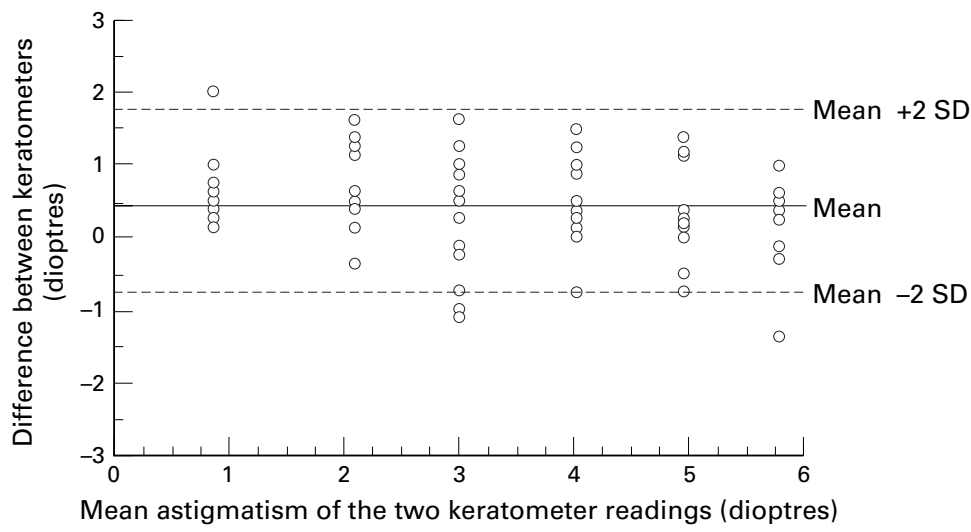

Figure 6 Limits of agreement between measurements from the keratoscopic astigmatic ruler and the Bausch $\mathcal{E}$ Lomb keratometer.

technique for the B\&L keratometer. Each button was measured in a predetermined randomised masked fashion four times by both observers. The horizontal measurement was subtracted from the vertical measurement to give the magnitude of astigmatism.

To assess the accuracy of the "sight" of the KAR, we measured the distance at which it was held in front of five patients' eyes. One observer would align the KAR while the other would measure the distance from the corneal apex to the KAR. Each patient had the ruler placed in front of the eye five times by each observer and a separate measurement was taken without feedback to the user of the KAR.

The measurements were analysed as means and standard deviations. The standard error of measurement (SEM), intraclass coefficient of reliability, coefficient of variance, and interobserver reliability coefficients were calculated by analysis of variance as described by Fliess. ${ }^{5}$ The coefficient of repeatability was determined as $2 \times$ SEM $^{6}$ Bland-Altman plots were used to assess visually the limits of agreement of measurement between the two keratometers. ${ }^{7}$

\section{Results}

The mean distance that the KAR was held from each patient's eye by each observer is shown in Table 1 . The mean distance the KAR was held from the eye from all measurements was $20.19 \mathrm{~mm}$ (95\% confidence interval (CI) 20.03 to 20.33). There was no difference between the novice and experienced user $(\mathrm{p}=$ 0.499, ANOVA).

The mean measurement and standard deviation for each button by each observer is shown in Table 2. There was no significant difference between the observers using the KAR ( $p=1.0$, ANOVA) or the B\&L ( $p=0.953$, ANOVA). However, there was a slight systematic error in the measurements using the B\&L, observer 1 under-reading the astigmatism by a mean of $0.41 \mathrm{D}$.

The means of the combined readings are shown graphically in Figures 2 and 3. The reliability calculations show that the keratometers compare favourably (Tables 2 and 3). The standard error of measurement (SEM) was $0.59 \mathrm{D}$ for the KAR, and $0.31 \mathrm{D}$ for the B\&L. The KAR had an intraclass correlation coefficient of reliability (R) of 0.90 (95\% CI of R, $>0.79)$, and the $\mathrm{B} \& \mathrm{~L}$ had an $\mathrm{R}$ of $0.97(95 \%$ CI, >0.94). The coefficient of repeatability (that is, the $95 \% \mathrm{CI}$ of test-retest) was plus or minus $0.83 \mathrm{D}$ for the KAR, and plus or minus $0.77 \mathrm{D}$ for the B\&L. The coefficient of variance was 0.405 for the KAR, and 0.091 for the $\mathrm{B} \& \mathrm{~L}$. The plots of the limits of agreement for the KAR, B\&L, and the KAR compared with the B\&L are shown in Figures 4, 5, and 6. The interobserver reliability (using a random effects model to generalise to the total population of observers) for the KAR was 0.898 (95\% CI $>0.78$ ), and for the B\&L, 0.975 (95\% CI $>0.66)$.

\section{Discussion}

The B\&L keratometer is widely used in clinical practice providing two corneal radii of curvature $90^{\circ}$ apart in dioptres and has a scale increment of $0.125 \mathrm{D}$. The KAR is useful for semiquantitative assessment of the relative corneal astigmatism at the slit-lamp or through the operating microscope at the time of surgery. Although it has a larger scale increment of $1 \mathrm{D}$, it was shown by our measurements to have good reliability and reproducibility, as well as being accurate within the increment of its scale. It compares favourably with the B\&L keratometer for the assessment of astigmatism.

We found no significant difference between the measurements taken by the experienced user compared with those taken by the novice. This suggests that once the method of use is understood, any user can reasonably expect to make reliable measurements. 
There may have been some difficulties in determining the astigmatism for the 1 and $2 \mathrm{D}$ buttons possibly because of some irregularity in the buttons' astigmatic cut. The differences in the KAR and B\&L measurements for the 1 and $2 \mathrm{D}$ buttons (Fig 3) may also be because the KAR only has a $1 \mathrm{D}$ increment, tending to "round up" the measurement.

Surgical keratometers are useful for the assessment of corneal astigmatism induced at the time of surgery. ${ }^{89}$ Knowledge of the magnitude of astigmatism provides the surgeon with instant feedback about the effect of surgical manoeuvres. Large astigmatic errors following cataract surgery were shown to be reduced by the use of a surgical keratometer. ${ }^{9}{ }^{10}$ A recent controlled trial demonstrated the value of intraoperative keratometry in the reduction of postcorneal graft astigmatism. ${ }^{1}$ Unlike microscope mounted keratometers, the KAR is portable, inexpensive, and easy to use. ${ }^{3}$ The KAR can also be used at the slit lamp during suture adjustment procedures,${ }^{11}$ allowing rapid quantification of the changes induced, without requiring the patient to shift from slit lamp to keratometer. The cornea often produces distorted mires on keratometry in the early postoperative period which precludes measurement of corneal curvature; however, we find the KAR is often a useful alternative in these circumstances.
In conclusion, we found the KAR a simple and useful adjunct to our clinical practice that requires little experience to take reliable and accurate measurements of corneal astigmatism.

NM and PL have a proprietary interest in the keratoscopic astigmatic ruler.

Presented in part at the ARVO meeting 1993.

1 Serdarvic ON, Renard GJ, Pouliquen Y. Randomised clinical trial comparing astigmatism and visual rehabilitation after penetrating keratoplasty with and without intraafter penetrating keratoplasty with and without intra9 .

2 Lindsay PA, Morlet N. A quantitative scale for the Barrett keratoscope. Aust NZ F Ophthalmol 1992;20:47-50.

3 Morlet N, Lindsay PA. Intraoperative semi-quantitative keratometry using a keratoscopic astigmatic ruler. $\mathcal{F}$ Cataract Refract Surg 1995;21:616-9.

4 Morlet N, Lindsay PA, Cooke P. A comparison of two semiquantitative surgical keratometers: the modified Hyde ruler and the Barrett keratoscope with the astigmatic dial. Ophthalmic Surg 1994;25:144-9.

5 Fleiss JL. The design and analysis of clinical experiments. Chapter 1. Chichester: John Wiley, 1986.

6 Arditi A, Cagenello R. On the statistical reliability of letterchart visual acuity measurements. Invest Ophthalmol Vis Sci 1993;34:120-9.

7 Bland JM, Altman DG. Statistical methods for the assessing agreement between two methods of clinical measurement. Lancet 1986;i:307-10.

8 Masket S. Correlation between intra-operative and early postoperative keratometry. $\mathcal{7}$ Cataract Refract Surg 1988;14: postoperati

9 Amoils SP. Intraoperative keratometry with the oval comparator (astigmometer). Br F Ophthalmol 1986;70:708-11.

10 Morlet N. Clinical utility of the Barrett keratoscope with astigmatic dial. Ophthalmic Surg 1994;25:150-3.

11 Allan BDS, Barrett GD. Qualitative keratoscopy at the slitlamp in postkeratoplasty suture adjustment. $\mathcal{f}$ Cataract Refract Surg 1993;19:817. 